\title{
Phonon Collapse and Second-Order Phase Transition in Thermoelectric SnSe
}

\author{
Unai Aseginolaza, ${ }^{1,2,3}$ Raffaello Bianco, ${ }^{4,5,6}$ Lorenzo Monacelli, ${ }^{4}$ Lorenzo Paulatto, ${ }^{7}$ Matteo Calandra, ${ }^{8}$ \\ Francesco Mauri, ${ }^{4,5}$ Aitor Bergara, ${ }^{1,2,9}$ and Ion Errea ${ }^{2,3}$ \\ ${ }^{1}$ Centro de Física de Materiales CFM, CSIC-UPV/EHU, Paseo Manuel de Lardizabal 5, \\ 20018 Donostia, Basque Country, Spain \\ ${ }^{2}$ Donostia International Physics Center (DIPC), Manuel Lardizabal pasealekua 4, 20018 Donostia, Basque Country, Spain \\ ${ }^{3}$ Fisika Aplikatua 1 Saila, University of the Basque Country (UPV/EHU), \\ Europa Plaza 1, 20018 Donostia, Basque Country, Spain \\ ${ }^{4}$ Dipartimento di Fisica, Università di Roma La Sapienza, Piazzale Aldo Moro 5, I-00185 Roma, Italy \\ ${ }^{5}$ Graphene Labs, Fondazione Istituto Italiano di Tecnologia, Via Morego, I-16163 Genova, Italy \\ ${ }^{6}$ Department of Applied Physics and Material Science, Steele Laboratory, California Institute of Technology, \\ Pasadena, California 91125, USA \\ ${ }^{7}$ IMPMC, UMR CNRS 7590, Sorbonne Universités-UPMC Univ. Paris 06, \\ MNHN, IRD, 4 Place Jussieu, F-75005 Paris, France \\ ${ }^{8}$ Sorbonne Universités, CNRS, Institut des Nanosciences de Paris, UMR7588, F-75252 Paris, France \\ ${ }^{9}$ Departamento de Física de la Materia Condensada, University of the Basque Country (UPV/EHU), \\ 48080 Bilbao, Basque Country, Spain
}

(Received 19 July 2018; revised manuscript received 29 November 2018; published 22 February 2019)

\begin{abstract}
Since 2014 the layered semiconductor $\mathrm{SnSe}$ in the high-temperature $\mathrm{Cm} \mathrm{cm}$ phase is known to be the most efficient intrinsic thermoelectric material. Making use of first-principles calculations we show that its vibrational and thermal transport properties are determined by huge nonperturbative anharmonic effects. We show that the transition from the $\mathrm{Cm} \mathrm{cm}$ phase to the low-symmetry Pnma is a second-order phase transition driven by the collapse of a zone border phonon, whose frequency vanishes at the transition temperature. Our calculations show that the spectral function of the in-plane vibrational modes are strongly anomalous with shoulders and double-peak structures. We calculate the lattice thermal conductivity obtaining good agreement with experiments only when nonperturbative anharmonic scattering is included. Our results suggest that the good thermoelectric efficiency of SnSe is strongly affected by the nonperturbative anharmonicity.
\end{abstract}

DOI: 10.1103/PhysRevLett.122.075901

Thermoelectric materials can convert waste heat into electricity $[1,2]$. The thermoelectric efficiency of a material is measured by the dimensionless figure of merit $Z T=S^{2} \sigma T / \kappa$, where $S$ is the Seebeck coefficient, $\sigma$ the electrical conductivity, $T$ the temperature, and $\kappa=\kappa_{e}+\kappa_{l}$ the thermal conductivity, constituted by electronic $\kappa_{e}$ and lattice $\kappa_{l}$ contributions. The thermoelectric efficiency can be thus enhanced by decreasing the thermal conductivity while keeping a high power factor $S^{2} \sigma$. Materials have been doped [3-5] or nanostructured [6,7] in order to get a high power factor combined with a low thermal conductivity, yielding, i.e., $Z T \simeq 2.2$ in $\mathrm{PbTe}$ [8]. In the proximity to a phase transition $Z T$ may also soar, as in the case of $\mathrm{Cu}_{2} \mathrm{Se}$ [9]. Recently, however, Zhao et al. reported for SnSe [10] the highest thermoelectric figure of merit ever reached in a material without doping, material treatment, or without being sharply enhanced by the proximity to a phase transition: $Z T \simeq 2.6$ above $800 \mathrm{~K}$.

$\mathrm{SnSe}$ is a narrow gap semiconductor that crystallizes at room temperature in an orthorhombic Pnma phase. At $T \simeq$ $800 \mathrm{~K}$ [10-13] it transforms into a more symmetric basecentered orthorhombic $\mathrm{Cmcm}$ structure (see Fig. 1). The

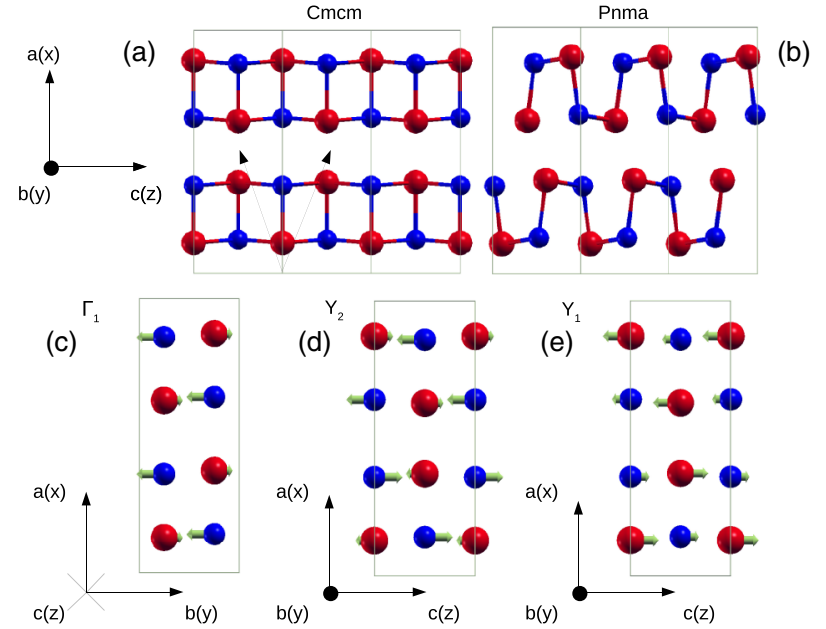

FIG. 1. The primitive lattice vectors of the $\mathrm{Cmcm}$ structure are $\mathbf{a}_{1}=(a / 2,0, c / 2), \mathbf{a}_{2}=(-a / 2,0, c / 2)$, and $\mathbf{a}_{3}=(0, b, 0)$, where $a$ (long axis), $b$, and $c$ are the lattice constants of the conventional cell. (a) $X Z$ face of the $C m c m$ structure. Primitive lattice vectors are denoted with arrows for convenience we show $\mathbf{a}_{1}$ and $-\mathbf{a}_{2}$. (b) $X Z$ face of the Pnma structure. (c) Atomic displacements of mode $\Gamma_{1}$. (d) Atomic displacements of mode $Y_{2}$. (e) Atomic displacements of mode $Y_{1}$. Sn atoms are red and Se blue. 
order of the transition is not clear: some works [10-12] claim it is a second-order transition and others it has a firstorder character [13]. A recent work [14] argues the transition occurs in two steps, where increasing temperature induces first a change in the lattice parameters that induces after a lattice instability. There is no inelastic scattering experiment so far for the high-temperature phase, which should show a prominent phonon collapse at the transition temperature if it belonged to the displacive second-order type [15-17].

The most interesting thermoelectric properties appear in the high-temperature phase, where the reduction of the electronic band gap increases the number of carriers providing a higher power factor, while the thermal conductivity remains very low [10]. The value of the intrinsic $\kappa_{l}$ of SnSe remains controversial, as the extremely low isotropic $0.3 \mathrm{~W} / \mathrm{mK}$ value at $800 \mathrm{~K}$ reported by Zhao et al. [10] could not be reproduced in other experiments, where a clear anisotropy is shown and the in-plane thermal conductivity is considerably larger [18-20]. The lattice thermal conductivity of the Pnma phase has been calculated from first principles solving the Boltzmann transport equation (BTE) using harmonic phonons and third order forceconstants (TOFCs) obtained perturbatively as derivatives of the Born-Oppenheimer energy surface [21,22]. The $\mathrm{Cmcm}$ phase has imaginary phonon frequencies in the harmonic approximation [14,22,23], as expected for the highsymmetry phase in a second-order transition [24-26], and it is stabilized by anharmonicity [14,22], hindering the calculation of $\kappa_{l}$ [22].

In this Letter, by performing ab initio calculations including anharmonicity at a nonperturbative level, we show that the phonon mode that drives the instability collapses at the transition temperature $T_{c}$ demonstrating that the transition is second order. Anharmonic effects are so large that the spectral function expected for some inplane modes deviates from the Lorentzian-like shape and shows broad peaks, shoulders, and satellite peaks, as in other monochalcogenides $[25,27]$. We calculate the lattice thermal conductivity of the $\mathrm{Cmcm}$ phase by combining the anharmonic phonon spectra with perturbative and nonperturbative TOFCs. We show here for the first time that nonperturbative anharmonic effects are not only crucial in the phonon spectra, but also in high-order force constants, which have a huge impact on the calculated thermal conductivity: $\kappa_{l}$ agrees with experiments [18] only with nonperturbative TOFCs.

The group/subgroup index of the $\mathrm{Cmcm} / \mathrm{Pnma}$ transition is 2, making a displacive second-order transition possible [28]. In this scenario, the transition temperature $T_{c}$ is defined as the temperature at which the second derivative of the free energy $F$ with respect to the order parameter $Q$ that transforms the structure continuously from the $\mathrm{Cmcm}$ phase $(Q=0)$ into the Pnma $(Q \neq 0)$ vanishes. As was already pointed out [12], symmetry [29,30] dictates that the amplitude of the transition is dominated by the distortion pattern associated to a nondegenerate mode $\left(Y_{1}\right)$ at the zone border $Y$ point with irreducible representation $Y_{2}^{+}$(see Fig. 1 for the distortion pattern). This means that $\partial^{2} F / \partial Q^{2}$ is proportional to the eigenvalue of the free energy Hessian matrix associated to this irreducible representation: $\omega_{Y_{1}}^{2}$.

In this work we calculate the free energy Hessian using the stochastic self-consistent harmonic approximation (SSCHA) [31,32], which is applied using ab initio density-functional theory (DFT) calculations within the Perdew-Burke-Ernzerhof (PBE) [33] or local density approximation (LDA) [34] parametrizations of the exchange-correlation functional (see Supplemental Material [35] for the details of the calculations [40-44]). The SSCHA is based on variational minimization of the free energy using a trial harmonic density matrix $\rho_{\mathcal{R}, \boldsymbol{\Phi}}$ parametrized by centroid positions $\mathcal{R}$ and force constants $\boldsymbol{\Phi}$ (bold symbols represent tensors in compact notation). The centroids $\mathcal{R}$ determine the most probable position of the atoms and $\boldsymbol{\Phi}$ is related to the amplitude of their fluctuations around $\mathcal{R}$. The free energy Hessian can be calculated as [32]

$$
\frac{\partial^{2} F}{\partial \mathcal{R} \partial \mathcal{R}}=\boldsymbol{\Phi}+\stackrel{(3)}{\mathbf{\Phi}} \mathbf{\Lambda}(0)[\mathbf{1}-\stackrel{(4)}{\mathbf{\Phi}} \mathbf{\Lambda}(0)]^{-1} \stackrel{(3)}{\mathbf{\Phi}}
$$

where $\stackrel{(3)}{\boldsymbol{\Phi}}$ and $\stackrel{(4)}{\boldsymbol{\Phi}}$ are third- and fourth-order nonperturbative force constants obtained as quantum averages calculated with $\rho_{\mathcal{R}, \boldsymbol{\Phi}}: \stackrel{(n)}{\boldsymbol{\Phi}}=\left\langle\partial^{n} V / \partial \mathbf{R}^{n}\right\rangle_{\rho_{\mathcal{R}, \Phi}}$. The $\stackrel{(n)}{\boldsymbol{\Phi}}$ force constants are generally different from the $\stackrel{(n)}{\Phi}$ perturbative ones obtained as derivatives of the Born-Oppenheimer potential $V$ at the minimum: $\boldsymbol{\phi}=\left[\partial^{n} V / \partial \mathbf{R}^{n}\right]_{0} . \boldsymbol{\Lambda}(0)$ in Eq. (1) is a function of the $\tilde{\Omega}_{\mu}$ SSCHA frequencies and polarization vectors obtained diagonalizing $\Phi_{a b} / \sqrt{M_{a} M_{b}}$, with $M_{a}$ the atomic mass ( $a$ labels both an atom and Cartesian index). The $\omega_{\mu}$ frequencies obtained instead from the free energy Hessian after diagonalizing $\left[\left(\partial^{2} F\right) /\left(\partial \mathcal{R}_{a} \partial \mathcal{R}_{b}\right)\right] / \sqrt{M_{a} M_{b}}$, e.g., $\omega_{Y_{1}}$, can be interpreted as the static limit of the (4)

physical phonons [32]. The contribution of $\boldsymbol{\Phi} \boldsymbol{\Lambda}$ is negligible with respect to the identity matrix [35] and thus it is neglected throughout.

The calculated temperature dependence of $\omega_{Y_{1}}^{2}$ is shown in Fig. 2 for LDA and PBE for two different lattice volumes in each case. In all cases $\omega_{Y_{1}}^{2}$ is positive at high temperatures, but it rapidly decreases with lowering the temperature, vanishing at $T_{c}$. This phonon collapse is consistent with a second-order phase transition between the Pnma and $C m \mathrm{~cm}$. We check that a SSCHA calculation at $T>T_{c}$ $(T=800 \mathrm{~K})$ starting from the relaxed low-symmetry Pnma phase (relaxed at DFT static level) yields the high-symmetry $\mathrm{Cmcm}$ atomic positions for the $\mathcal{R}$ 

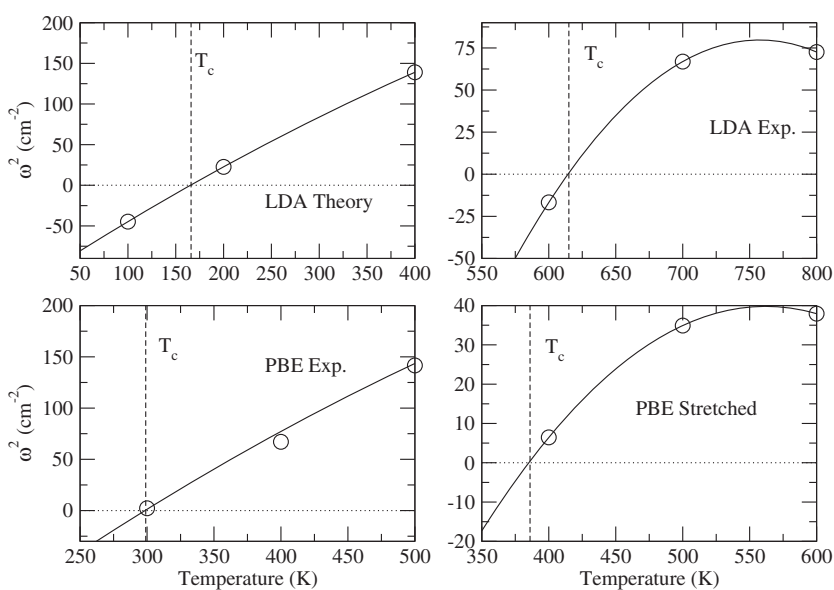

FIG. 2. $\omega_{Y_{1}}^{2}$ as a function of temperature within LDA and PBE approximations for different lattice volumes (circles). In the LDA we compare the results obtained with the theoretical and experimental [10] lattice parameters. In the PBE calculation we present the results for the experimental lattice parameters and a stretched unit cell (see Table I for the lattice parameters). The solid lines correspond to a polynomial fit.

centroids. Thus, the Pnma is not a local minimum of the free energy above $T_{c}$, ruling out the first-order transition. Our result disagrees with the conclusions drawn in Ref. [14]. First, because at the $T_{c}$ calculated in Ref. [14], which is estimated by comparing the free energies of the two structures, the $Y_{1}$ mode of the Cmcm phase is stable, which implies this phase is a local minimum at $T_{c}$, and, thus, the transition is of first-order type [14]. And second, because it is argued [14] that the instability at $Y$ is produced by a slight change in the inplane lattice parameters induced by temperature (from $c / b>1$ to $c / b<1$ ), which makes the transition a twostep process. We do not see this sudden appearance of the instability [35].

The obtained transition temperature strongly depends on the exchange-correlation functional and the volume, as it occurs in similar monochalcogenides [25]. Within LDA $T_{c}$ ranges between $168 \mathrm{~K}$ with theoretical lattice parameters and $616 \mathrm{~K}$ with experimental lattice parameters [10]. Within PBE $T_{c}$ barely changes between the experimental and theoretical lattice parameters. We attribute this result to the fact that the in-plane lattice parameters $b$ and $c$ are in perfect agreement with the experimental results within PBE, while LDA clearly underestimates them. The theoretical lattice parameters are estimated neglecting vibrational contributions to the free energy. In order to estimate the role of the thermal expansion, we calculate the stress tensor including vibrational contributions at the anharmonic level [45]. The in-plane contribution of the stress tensor calculated at the temperature closest to $T_{c}, P_{z z}$, shows that both theoretical LDA and PBE lattices should be stretched. Within LDA it is clear that stretching the lattice increases $T_{c}$. Within PBE, when we take a stretched lattice
TABLE I. Experimental [10] and theoretical (DFT at static level) LDA and PBE lattice parameters used in this work. The stretched cell used in some calculations is also given. $a, b$, and $c$ lattice parameters are given in Bohr length units $\left(a_{0}\right)$ and the three components of the stress tensor in GPa units. The pressure is calculated including vibrational terms at an anharmonic level at the following temperatures for each case: $200 \mathrm{~K}$ (LDA theory), $600 \mathrm{~K}$ (LDA Exp.), $400 \mathrm{~K}$ (PBE Exp.), $400 \mathrm{~K}$ (PBE theory), and $400 \mathrm{~K}$ (PBE stretched).

\begin{tabular}{lcccrrr}
\hline \hline & $a$ & $b$ & $c$ & $P_{x x}$ & $P_{y y}$ & $P_{z z}$ \\
\hline LDA theory & 21.58 & 7.90 & 7.90 & 0.4 & 0.6 & 0.7 \\
LDA exp. & 22.13 & 8.13 & 8.13 & -1.1 & -2.0 & -2.2 \\
PBE theory & 22.77 & 8.13 & 8.13 & 0.5 & 1.1 & 1.0 \\
PBE exp. & 22.13 & 8.13 & 8.13 & 1.8 & 1.3 & 1.2 \\
PBE stretched & 23.48 & 8.27 & 8.27 & -0.3 & -0.7 & -0.7 \\
\hline \hline
\end{tabular}

to reduce $P_{z z}, T_{c}$ increases from 299 to $387 \mathrm{~K}$. In all cases the other in-plane component of the stress tensor, $P_{y y}$, is very similar to $P_{z z}$. The LDA transition temperature with the experimental lattice parameters yields the transition temperature in closest agreement with experiments $\left(T_{c} \simeq 800 \mathrm{~K}[10-13,46]\right)$. The underestimation of the transition temperature may be due to the approximated exchange-correlation or the finite supercell size taken for the SSCHA.

The predicted phonon collapse should be measurable by inelastic neutron scattering (INS) experiments. INS experiments [46] show a softening of a zone-center optical mode of the Pnma phase upon heating, which is consistent with the condensation of the $Y_{1}$ mode after the transition. By making use of a dynamical ansatz [32], we calculate the mode-projected phonon anharmonic self-energy [35] $\Pi_{\mu}(\mathbf{q}, \omega)$, from which we obtain the phonon spectral function:

$$
\begin{aligned}
& \sigma(\mathbf{q}, \omega) \\
& =\frac{1}{\pi} \sum_{\mu} \frac{-\omega \operatorname{Im}_{\mu}(\mathbf{q}, \omega)}{\left[\omega^{2}-\tilde{\Omega}_{\mu}^{2}(\mathbf{q})-\operatorname{Re} \Pi_{\mu}(\mathbf{q}, \omega)\right]^{2}+\left[\operatorname{Im} \Pi_{\mu}(\mathbf{q}, \omega)\right]^{2}} .
\end{aligned}
$$

Peaks in $\sigma(\mathbf{q}, \omega)$ represent experimental phonon excitations. Replacing $\Pi_{\mu}(\mathbf{q}, \omega) \rightarrow \Pi_{\mu}\left(\mathbf{q}, \tilde{\Omega}_{\mu}(\mathbf{q})\right)$ in Eq. (2) the Lorentzian approximation is recovered, in which each peak is represented with a Lorentzian function centered at $\Omega_{\mu}(\mathbf{q})=\sqrt{\tilde{\Omega}_{\mu}^{2}(\mathbf{q})+\operatorname{Re}_{\mu}\left(\mathbf{q}, \tilde{\Omega}_{\mu}(\mathbf{q})\right)}$ with a linewidth proportional to $\operatorname{Im} \Pi_{\mu}\left(\mathbf{q}, \tilde{\Omega}_{\mu}(\mathbf{q})\right)$ [47].

Figure 3 compares the harmonic phonon spectrum with the anharmonic one in the Lorentzian approximation obtained at $800 \mathrm{~K}$ within LDA in the experimental lattice (the results below are also obtained within the LDA in the experimental lattice). The anharmonic correction is large 


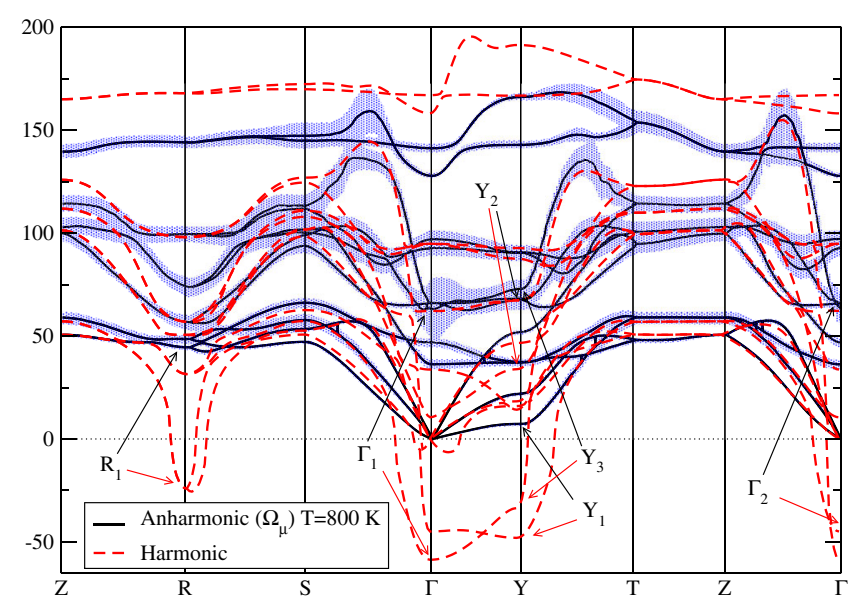

FIG. 3. Harmonic and anharmonic phonons in the Lorentzian approximation $\left[\Omega_{\mu}(\mathbf{q})\right]$. The length of the bars corresponds to the linewidth (full length of the line is the full width at half maximum). The calculations are done within LDA in the (3)

experimental structure using $\boldsymbol{\Phi}$ at 800 and $\tilde{\Omega}_{\mu}(\mathbf{q})$ at $800 \mathrm{~K}$.

for most of the modes across the Brillouin zone. Within the harmonic approximation, there are five unstable modes: two $\left(\Gamma_{1}, \Gamma_{2}\right)$ at $\Gamma$, two $\left(Y_{1}, Y_{3}\right)$ at $Y$, and one $\left(R_{1}\right)$ at $R$. The instabilities at $\Gamma$ would cause ferroeletric transitions $[22,48]$, but they suffer an anharmonic renormalization that prevents it. $Y_{3}$ and $R_{1}$ are also stabilized by anharmonic effects. The $Y_{1}$ mode, however, remains unstable at $600 \mathrm{~K}$ and it is stabilized after the transition [see Figs. 4(a) and 4(b)].

In highly anharmonic materials $[25,27,47,49,50]$, the spectral functions show broad peaks, shoulders, and satellite peaks, strongly deviating from the Lorentzian picture. In Fig. 4 we show the spectral function keeping the full frequency dependence on the self-energy, without assuming the Lorentzian line shape. The spectral function clearly reproduces the collapse of the $Y_{1}$ mode at the transition temperature. The calculated spectral functions show that the strong anharmonicity present on the phonon frequency renormalization is also reflected on the spectral function. The strongly anharmonic features specially affect in-plane modes in the $25-75 \mathrm{~cm}^{-1}$ energy range. For instance, at the $\Gamma$ point the $\Gamma_{1}$ mode, which describes a vibration along the in-plane $y$ axis in opposite direction for the Sn and Se atoms (see Fig. 1) and is stabilized by anharmonicity, shows a double peak structure and a broad shoulder [see Fig. 4(e)]. The mode that describes the same vibration $\left(\Gamma_{2}\right)$ but in the other in-plane $z$ direction also shows a complex non-Lorentzian shape. The overall $\sigma(\mathbf{q}=\Gamma, \omega)$ consequently has a broad shoulder at $\simeq 25 \mathrm{~cm}^{-1}$ as marked in Fig. 4(c), which is less acute as temperature increases. At the $Y$ point there are also two modes, $Y_{2}$, whose eigenvector is plotted in Fig. 1, and $Y_{3}$, which describes the same displacement but in the other $y$ in-plane direction, that show a strongly anharmonic nonLorentzian shape. The modes with complex line shapes are those that show the largest linewidth in the Lorentzian limit (see Fig. 3). These modes have strongly anomalous spectral functions and large linewidths because they can easily scatter with an optical mode close in energy and an acoustic mode close to $\Gamma$. We identify this by directly analyzing which phonon triplets contribute more to the linewidth. It is interesting to remark that if $\Pi_{\mu}(\mathbf{q}, \omega)$ is calculated by (3) (3)

substituting $\boldsymbol{\Phi}$ by $\boldsymbol{\phi}$, the anomalies of these modes become weaker [35]. This underlines that in the $\mathrm{Cmcm}$ phase the third-order derivatives of $V$ are not sufficient to calculate the phonon linewidths and that higher order terms are (3) important, which are effectively captured by $\boldsymbol{\Phi}$.
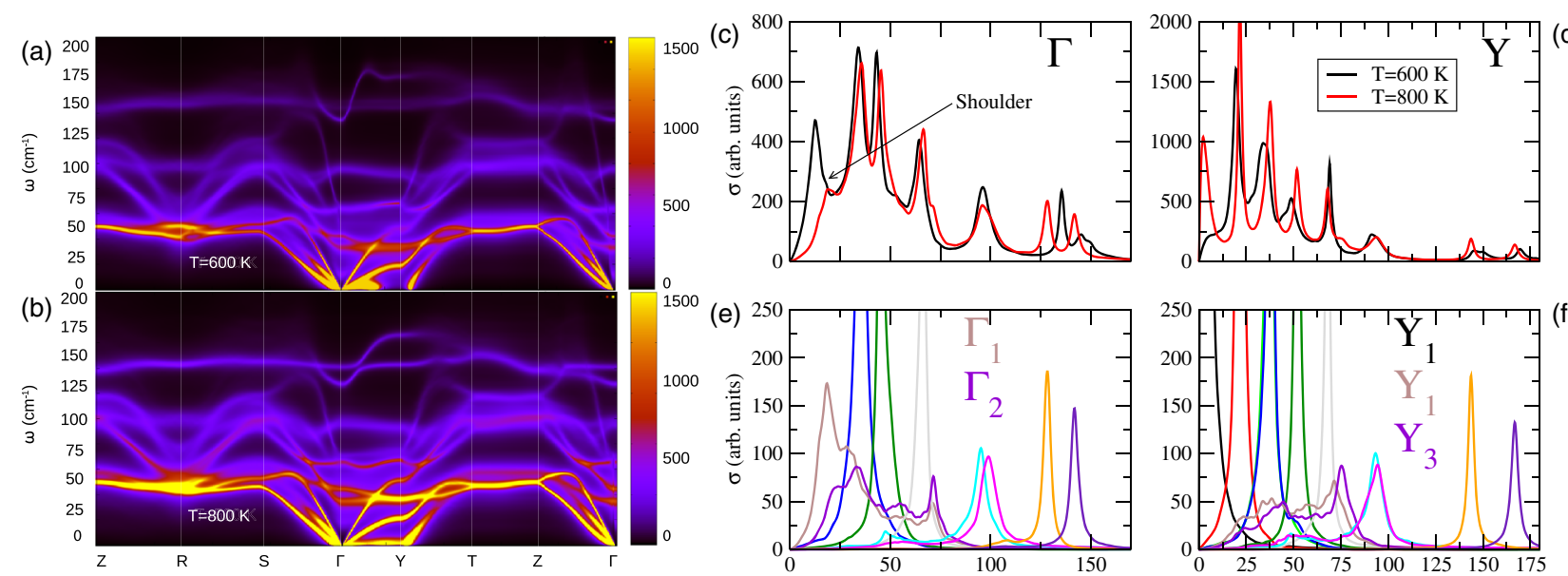

(d)
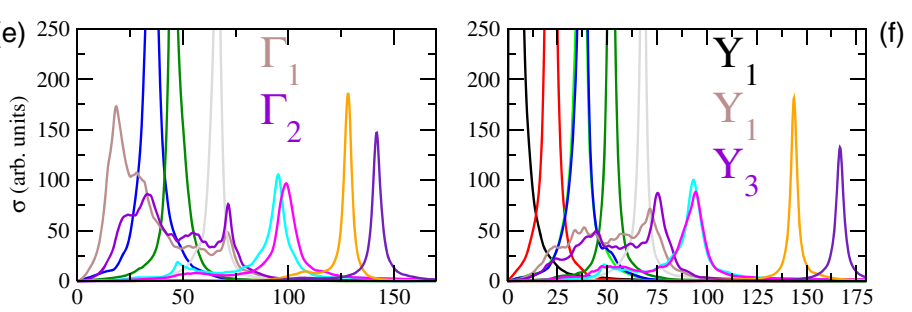

(3)

FIG. 4. Spectral function of SnSe in the $\mathrm{Cmcm}$ phase calculated at (a) 600 and (b) $800 \mathrm{~K}$ using $\boldsymbol{\Phi}$ at the corresponding temperature. The spectral function at the (c) $\Gamma$ and (d) $Y$ points at 600 and $800 \mathrm{~K}$. The contribution of each mode to the spectral function is also shown at the $\Gamma$ point (e) and the $Y$ point (f) at $800 \mathrm{~K}$. Different colors correspond to different modes. All the calculations are performed within LDA in the experimental structure. In each case we use $\tilde{\Omega}_{\mu}(\mathbf{q})$ calculated at the same temperature as $\stackrel{(3)}{\mathbf{\Phi}}$. 


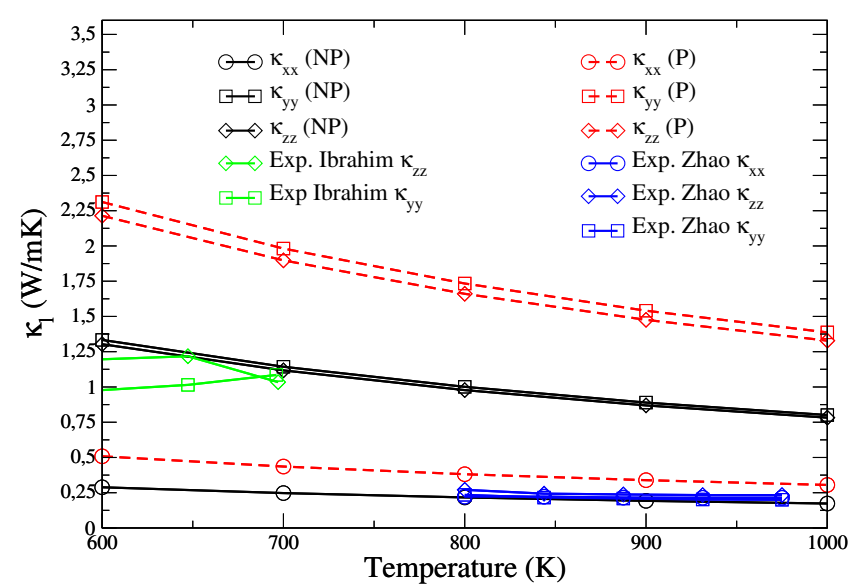

FIG. 5. Lattice thermal conductivity of SnSe calculated with perturbative $\stackrel{(3)}{\boldsymbol{\phi}}(P)$ and nonperturbative $\stackrel{(3)}{\boldsymbol{\Phi}}(\mathrm{NP})$ at $800 \mathrm{~K}$ compared to the experiments by Ibrahim et al. [18] and Zhao et al. [10]. We use the $\tilde{\Omega}_{\mu}(\mathbf{q})$ phonon frequencies calculated at $800 \mathrm{~K}$ at all temperatures. Calculations are performed within LDA using the experimental structure. Different volumes or exchange-correlation functionals give consistent results [35].

In Fig. 5 we present the lattice thermal conductivity calculated with the SSCHA frequencies $\left(\tilde{\Omega}_{\mu}(\mathbf{q})\right)$ and nonperturbative TOFCs $(\stackrel{(\boldsymbol{\Phi})}{)}$. For comparison we also calculate $\kappa_{l}$ substituting $\stackrel{(3)}{\boldsymbol{\Phi}}$ by $\stackrel{(3)}{\boldsymbol{\phi}}$. The calculation is performed solving the BTE assuming the single-mode relaxation time approximation (SMA). The thermal conductivity of $\mathrm{SnSe}$ is very low, mainly because the contribution of optical modes is strongly suppressed by the large anharmonicity and the contribution of acoustic modes is also reduced due to the large scattering among themselves and with the $\Gamma_{1}$ mode. We compare our results with the values obtained by Zhao et al. [10] above the transition at $800 \mathrm{~K}$. We also include in the figure the results obtained by Ibrahim et al. [18] above $600 \mathrm{~K}$ (only the in-plane $\kappa_{l}$ is reported at these temperatures) in the Pnma phase. Even if the results belong to different phases, comparing our calculations for the $\mathrm{Cmcm}$ phase with those obtained in the latter work is insightful because the thermal conductivity of these two phases is very similar close to the transition [35], as expected in a second-order phase transition. Though direct comparison should be taken carefully for this reason, the lattice thermal conductivity is in better agreement with experimental results using $\stackrel{(3)}{\boldsymbol{\Phi}}$ instead of $\stackrel{(3)}{\boldsymbol{\phi}}$, which overestimates the lattice thermal conductivity along the in-plane directions. This is consistent with the larger phonon linewidths obtained with the nonperturbative TOFCs. The agreement for the in-plane $\kappa_{y y} \sim \kappa_{z z}$ with the measurements by Ibrahim et al. [18] is good in the nonperturbative limit, contrary to previous calculations that underestimate it [22]. The calculated out-of-plane $\kappa_{x x}$ is also in good agreement with the results by Zhao et al. [10], but we find that their ultralow results for the in-plane $\kappa_{l}$, in contradiction with the values in Ref. [18] obtained for the low-symmetry phase close to the transition, are underestimated. These results suggest that the thermal conductivity measured by Zhao et al. may have nonintrinsic effects as it has already been pointed out [51].

In conclusion, we show that the vibrational properties of $\mathrm{SnSe}$ in the $\mathrm{Cmcm}$ phase are dominated by huge nonperturbative anharmonic effects. We show how the collapse of the $Y_{1}$ mode is responsible for the second-order phase transition. The calculated transition temperature is volume and functional dependent. The spectral functions of inplane modes are characterized by anomalous features deviating from the Lorentzian-like shape. These results will be crucial to interpret future INS experiments for the high-temperature phase. The calculated in-plane thermal conductivity is in good agreement with the experiments by Ibrahim et al. [18], but not with those by Zhao et al. [10] which show low anisotropy. These results suggest that the isotropic ultralow values by Zhao et al. could be the observation of a nonintrinsic property. Our results show for the first time that the inclusion of nonperturbative effects is crucial for obtaining third-order force constants that yield a lattice thermal conductivity in agreement with experiments.

The authors acknowledge fruitful discussions with $\mathrm{O}$. Delaire. Financial support was provided by the Spanish Ministry of Economy and Competitiveness (FIS201676617-P), the Department of Education, Universities and Research of the Basque Government and the University of the Basque Country (IT756-13). U. A. is also thankful to the Material Physics Center for support. Computer facilities were provided by the Donostia International Physics Center (DIPC), the Spanish Supercomputing Network (FI-2017-20007), and PRACE (2017174186).

[1] H. J. Goldsmid, Introduction to Thermoelectricity (Springer, New York, 2010), pp. 1-7.

[2] K. Behnia, Fundamentals of Thermoelectricity (OUP, Oxford, 2015).

[3] G.-H. Kim, L. Shao, K. Zhang, and K. P. Pipe, Nat. Mater. 12, 719 (2013).

[4] Y. Pei, A. D. LaLonde, N. A. Heinz, X. Shi, S. Iwanaga, H. Wang, L. Chen, and G. J. Snyder, Adv. Mater. 23, 5674 (2011).

[5] J. P. Heremans, V. Jovovic, E. S. Toberer, A. Saramat, K. Kurosaki, A. Charoenphakdee, S. Yamanaka, and G. J. Snyder, Science 321, 554 (2008).

[6] C. J. Vineis, A. Shakouri, A. Majumdar, and M. G. Kanatzidis, Adv. Mater. 22, 3970 (2010).

[7] A. Minnich, M. Dresselhaus, Z. Ren, and G. Chen, Energy Environ. Sci. 2, 466 (2009).

[8] K. F. Hsu, S. Loo, F. Guo, W. Chen, J. S. Dyck, C. Uher, T. Hogan, E. K. Polychroniadis, and M. G. Kanatzidis, Science 303, 818 (2004). 
[9] H. Liu, X. Yuan, P. Lu, X. Shi, F. Xu, Y. He, Y. Tang, S. Bai, W. Zhang, L. Chen et al., Adv. Mater. 25, 6607 (2013).

[10] L.-D. Zhao, S.-H. Lo, Y. Zhang, H. Sun, G. Tan, C. Uher, C. Wolverton, V.P. Dravid, and M. G. Kanatzidis, Nature (London) 508, 373 (2014).

[11] K. Adouby, Z. Kristallogr. 213, 343 (1998).

[12] T. Chattopadhyay, J. Pannetier, and H. Von Schnering, J. Phys. Chem. Solids 47, 879 (1986).

[13] H. G. Von Schnering and H. Wiedemeier, Z. Kristallogr. Cryst. Mater. 156, 143 (1981).

[14] A. Dewandre, O. Hellman, S. Bhattacharya, A. H. Romero, G. K. H. Madsen, and M. J. Verstraete, Phys. Rev. Lett. 117, 276601 (2016).

[15] M. Holt, P. Zschack, H. Hong, M. Y. Chou, and T.-C. Chiang, Phys. Rev. Lett. 86, 3799 (2001).

[16] F. Weber, S. Rosenkranz, J.-P. Castellan, R. Osborn, R. Hott, R. Heid, K.-P. Bohnen, T. Egami, A. H. Said, and D. Reznik, Phys. Rev. Lett. 107, 107403 (2011).

[17] C. D. O’Neill, D. A. Sokolov, A. Hermann, A. Bossak, C. Stock, and A. D. Huxley, Phys. Rev. B 95, 144101 (2017).

[18] D. Ibrahim, J.-B. Vaney, S. Sassi, C. Candolfi, V. Ohorodniichuk, P. Levinsky, C. Semprimoschnig, A. Dauscher, and B. Lenoir, Appl. Phys. Lett. 110, 032103 (2017).

[19] S. Sassy, C. Candolfi, J.-B. Vaney, V. Ohorodniichuk, P. Masschelein, A. Dauscher, and B. Lenoir, Appl. Phys. Lett. 104, 212105 (2014).

[20] C.-L. Cheng, H. Wang, Y.-Y. Chen, T. Day, and G. J. Snyder, J. Mater. Chem. A 2, 11171 (2014).

[21] J. Carrete, N. Mingo, and S. Curtarolo, Appl. Phys. Lett. 105, 101907 (2014).

[22] J. M. Skelton, L. A. Burton, S. C. Parker, A. Walsh, C.-E. Kim, A. Soon, J. Buckeridge, A. A. Sokol, C. R. A. Catlow, A. Togo et al., Phys. Rev. Lett. 117, 075502 (2016).

[23] H. Yu, S. Dai, and Y. Chen, Sci. Rep. 6, 26193 (2016).

[24] M. Iizumi, Y. Hamaguchi, K. F. Komatsubara, and Y. Kato, J. Phys. Soc. Jpn. 38, 443 (1975).

[25] G. A. S. Ribeiro, L. Paulatto, R. Bianco, I. Errea, F. Mauri, and M. Calandra, Phys. Rev. B 97, 014306 (2018).

[26] M. Jian, M. Trigo, S. Saviíc, S. Fahy, É. Murray, C. Bray, J. Clark, T. Henighan, M. Kozina, M. Chollet et al., Nat. Commun. 7, 12291 (2016).

[27] C. W. Li, O. Hellam, J. Ma, A. F. May, H. B. Cao, X. Chen, A. D. Christianson, G. Ehlers, D. J. Singh, B. C. Sales, and O. Delaire, Phys. Rev. Lett. 112, 175501 (2014).

[28] J.-C. Toledano and P. Toledano, The Landau Theory of Phase Transitions: Application to Structural, Incommensurate,
Magnetic and Liquid Crystal Systems (World Scientific, Singapore, 1987).

[29] D. Orobengoa, C. Capillas, M. I. Aroyo, and J. M. Perez-Mato, J. Appl. Crystallogr. 42, 820 (2009).

[30] J. M. Perez-Mato, D. Orobengoa, and M. I. Aroyo, Acta Crystallogr. Sect. A 66, 558 (2010).

[31] I. Errea, M. Calandra, and F. Mauri, Phys. Rev. B 89, 064302 (2014).

[32] R. Bianco, I. Errea, L. Paulatto, M. Calandra, and F. Mauri, Phys. Rev. B 96, 014111 (2017).

[33] J. P. Perdew, K. Burke, and M. Ernzerhof, Phys. Rev. Lett. 77, 3865 (1996).

[34] J. P. Perdew and A. Zunger, Phys. Rev. B 23, 5048 (1981).

[35] See Supplemental Material at http://link.aps.org/ supplemental/10.1103/PhysRevLett.122.075901 for more detailed analysis of crystal structure, theory, and calculation methods, which includes Refs. [36-39].

[36] G. Kresse and J. Hafner, J. Phys. Condens. Matter 6, 8245 (1994).

[37] P. E. Blöchl, Phys. Rev. B 50, 17953 (1994).

[38] N. Troullier and J. L. Martins, Phys. Rev. B 43, 1993 (1991).

[39] G. Fugallo, M. Lazzeri, L. Paulatto, and F. Mauri, Phys. Rev. B 88, 045430 (2013).

[40] S. Baroni, S. De Gironcoli, A. Dal Corso, and P. Giannozzi, Rev. Mod. Phys. 73, 515 (2001).

[41] L. Paulatto, F. Mauri, and M. Lazzeri, Phys. Rev. B 87, 214303 (2013)

[42] P. Giannozzi, S. Baroni, N. Bonini, M. Calandra, R. Car, C. Cavazzoni, D. Ceresoli, G. L. Chiarotti, M. Cococcioni, I. Dabo et al., J. Phys. Condens. Matter 21, 395502 (2009).

[43] P. Giannozzi et al., J. Phys. Condens. Matter 29, 465901 (2017).

[44] W. Li, J. Carrete, N. A. Katcho, and N. Mingo, Comput. Phys. Commun. 185, 1747 (2014).

[45] L. Monacelli, I. Errea, M. Calandra, and F. Mauri, Phys. Rev. B 98, 024106 (2018).

[46] C. W. Li, J. Hong, A. F. May, D. Bansal, S. Chi, T. Hong, G. Ehlers, and O. Delaire, Nat. Phys. 11, 1063 (2015).

[47] R. Bianco, I. Errea, M. Calandra, and F. Mauri, Phys. Rev. B 97, 214101 (2018).

[48] J. Hong and O. Delaire, arXiv:1604.07077.

[49] O. Delaire, J. Ma, K. Marty, A. F. May, M. A. McGuire, M.-H. Du, D. J. Singh, A. Podlesnyak, G. Ehlers, M. Lumsden et al., Nat. Mater. 10, 614 (2011).

[50] L. Paulatto, I. Errea, M. Calandra, and F. Mauri, Phys. Rev. B 91, 054304 (2015).

[51] P.-C. Wei, S. Bhattacharya, J. He, S. Neeleshwar, R. Podila, Y. Chen, and A. Rao, Nature (London) 539, E1 (2016). 